\title{
OBJETIVOS E ALGUMAS LIMITAÇÕES NA DESCRIÇÃO TERMODINÂMICA DE FASES E SISTEMAS ATRAVÉS DO MÉTODO CALPHAD
}

André Luiz Vasconcellos da Costa e Silva '

\section{Resumo}

A descrição das propriedades termodinâmica das fases e, conseqüentemente, dos sistemas termodinâmicos, através de equações é uma das bases do método CALPHAD. Entretanto, no processo de obtenção destas descrições através de equações, dificuldades são freqüentemente encontradas e muitas vezes sanadas de formas pouco proveitosas para o objetivo final. Assim, por exemplo, a tentativa de descrever com muita precisão (aparente) os dados disponíveis na literatura sobre determinada fase ou sistema tem levado a descrições CALPHAD que terminam não tendo utilidade prática além da geração de uma publicação ou de permitir reproduzir um diagrama de equilíbrio de fases. Neste trabalho são resumidos os objetivos do método CALPHAD e suas limitações, ressaltando alguns aspectos importantes na solução das dificuldades encontradas na descrição termodinâmica de fases e sistemas. Discutem-se as causas e as soluções para estas limitações (mesmo as praticamente inviáveis) e abordam-se os riscos de se adotar soluções "simplistas" para as situações encontradas.

Palavras-chave: Termodinâmica computacional; Modelamento matemático; Método CALPHAD; Diagrama de fase.

\section{OBJECTIVES AND SOME LIMITATIONS IN THE THERMODYNAMIC DESCRIPTION OF PHASES AND SYSTEMS USING THE CALPHAD APPROACH}

\begin{abstract}
\section{INTRODUÇÃO}

Diagramas de equilíbrio de fases são ferramentas essenciais na metalurgia e na ciência dos materiais. Embora a relação conceitual entre a termodinâmica e estes diagramas seja conhecida há mais de um século, durante muitas décadas praticamente a única forma de obtenção destes diagramas foi a realização de experimentos de equilíbrio. Duas principais dificuldades limitaram o uso das propriedades termodinâmicas para o cálculo de diagramas de equilíbrio, uma de ordem instrumental e outra conceitual. Embora no final da década de 1960 já se dispusesse de ferramental
\end{abstract}

Describing the thermodynamic functions of phases using equations is the basis of the CALPHAD method. However, during the process of obtaining these equations, some common difficulties can be found. Frequently the way these problems are solved is not the best if the maximum benefit of the CALPHAD method is desired. Thus, for instance attempts of reaching a very high apparent precision in describing the available data can lead to CALPHAD descriptions that have no additional use beyond being published or re-drawing the original phase diagram on which it is based. It the present work the objectives of the CALPHAD method, and its limitations are summarized, as well as some important issues in solving the difficulties encountered in describing the thermodynamics of a system. Ways of avoiding these pitfalls are presented and discussed as well as the risks resulting from not observing these precautions.

Key words: Computational thermodynamics; Mathematical modeling; CALPHAD; Phase diagrams.

computacional suficiente para tratar o problema e algumas iniciativas importantes relacionando dados termodinâmicos com diagramas de fases já existissem, persistia a dificuldade de tratar fases sobre as quais não era possível se obter dados experimentais diretamente, tipicamente fases metaestáveis. Um dos desenvolvimentos essenciais para alterar esta situação foram os lattice stability para fases metaestáveis calculados por Larry Kaufman na década de 1960 e o conseqüente desenvolvimento do método Computer CALculation of PHAse Diagrams (CALPHAD).

Um benefício adicional do método, que ampliou o interesse em sua aplicação, decorre da Segunda Lei da termodinâmica. ${ }^{(1)} \grave{A}$ medida que aumenta a ordem do sistema de interesse é menos provável que surjam novos compostos ou novas interações. Ou seja,

' Membro da ABM, Engenheiro Metalúrgico, PhD, Diretor Técnico do IBQN, Professor da EEIMVR-UFF, Volta Redonda - RJ (andre@metal.eeimvr.uff.br) 
as interações mais fortes entre constituintes, são interações binárias e, no máximo, interações ternárias. A partir daí, a contribuição da entropia domina a variação das propriedades nas misturas. $O$ efeito prático deste fato é que o comportamento de sistemas de ordem elevada (como, por exemplo, aços contendo seis ou mais elementos de liga) pode ser calculado a partir do conhecimento dos sistemas binários e ternários correspondentes, com alto nível de acerto.

Em função deste potencial e destas vantagens, associadas ao fato que, para produzir descrições matemáticas e calcular diagramas de equilíbrio de fases não é necessário investimento elevado de recursos em trabalho experimental, a pesquisa em termodinâmica computacional expandiu-se de forma explosiva. Infelizmente, estas facilidades permitem também que resultados de confiabilidade menor que a desejada sejam obtidos e publicados. Em alguns casos, por exemplo, o único critério para a avaliação da "qualidade" das descrições matemáticas tem sido sua capacidade de reproduzir os dados e "Figuras" empregados para sua obtenção. Naturalmente, quando o objetivo final é a descrição de sistemas mais complexos, estes testes podem ser insuficientes. $O$ objetivo deste trabalho é apresentar os objetivos do método CALPHAD e suas limitações, ressaltando alguns aspectos importantes na solução das dificuldades encontradas quando se busca descrições termodinâmicas de fases e sistemas. A solução destas dificuldades é essencial para a obtenção de descrições CALPHAD “úteis”. Este assunto tem sido tema de importantes discussões na comunidade que se dedica a esta técnica. ${ }^{(2)}$

\section{AS PROPRIEDADES TERMODINÂMICAS DOS ELEMENTOS PUROS}

\section{I As Propriedades das Estruturas Metaestáveis em Todas as Temperaturas: Lattice Stabilities}

Como muitos elementos são solúveis em quantidades consideráveis em estruturas nas quais não são estáveis (por exemplo, $\mathrm{Cr}(\mathrm{CCC})$ dissolve extensamente em $\mathrm{Ni}(\mathrm{CFC})^{(3)}$ ) é necessário conhecer as propriedades termodinâmicas destas fases metaestáveis, inacessíveis experimentalmente. Kaufman foi pioneiro no cálculo destes valores ${ }^{(4)}$ e, embora alguns valores propostos tenham sido contestados, foram a base para toda a metodologia CALPHAD hoje empregada.

Como a essência da aplicação prática do método CALPHAD consiste na combinação das descrições das funções termodinâmicas de sistemas de mais baixa ordem até a obtenção dos sistemas de alta ordem de interesse prático, é essencial que as propriedades dos elementos, em todas as estruturas consideradas possíveis nos sistemas de interesse sejam consistentes. Felizmente, desde 1991 há uma descrição destas propriedades, recomendada pelo Scientific Group Thermodata Europe (SGTE), que vem sendo adotada por praticamente todos os praticantes da área. ${ }^{(5)}$

\subsection{As Propriedades das Estruturas Metaestáveis em Algumas Faixas de Temperaturas: Extrapolações}

Da mesma forma, o método CALPHAD requer o conhecimento de propriedades de fases em faixas de temperatura muito distantes da região em que estas fases são estáveis. Um exemplo interessante é a fase líquida no sistema Fe-Zn. Para que o comportamento do sistema próximo às temperaturas solidus e liquidus na região rica em $Z n$ possa ser corretamente calculado, é necessário conhecer o comportamento do Fe líquido na região de temperatura de cerca de $400^{\circ} \mathrm{C}-500^{\circ} \mathrm{C}$, uma extrapolação considerável em relação à faixa onde experimentos são possíveis. Neste caso, também, a tendência tem sido adotar valores padronizados pelo SGTE.

\subsection{A Descrição Termodinâmica dos Elementos Puros e Outros End- members de Composição Definida}

Como discutido em outra publicação, para um elemento ou substância, as propriedades termodinâmicas de maior interesse (G, S, H, Cp) estão relacionadas entre si, de modo que só é necessário ter uma descrição matemática para uma delas, para se ter acesso a todas. ${ }^{(6)}$ A escolha, no método CALPHAD, recai sobre a energia livre de Gibbs, G. Esta função não tem um zero estabelecido. Embora seja convencional estabelecer que a entropia das substâncias puras é zero a $0 \mathrm{~K}$, a entalpia também não tem um zero estabelecido. O estado padrão escolhido para os elementos puros é, normalmente, a fase estável a 298, I5 K e 0, I MPa (I bar). Este estado é chamado de SER (standard element reference).

Antes da adoção da padronização proposta pelo SGTE para o SER era comum o emprêgo, como estado padrão, de uma das fases do elemento (normalmente a mais estável a 298, I5 K e 0, I MPa) a temperatura e pressão de cálculo. Assim, somente valores relativos (lattice stabilities) precisavam ser computados para o cálculo das fases em equilíbrio. Em que pese a vantagem da simplificação das descrições matemáticas empregadas para $\mathrm{G}$ neste enfoque, uma desvantagem significativa, que limita em muito seu emprego, é a perda de informação sobre o valor da entalpia em função da temperatura das diversas fases que impossibilita, por exemplo, a realização de balanços térmicos.

\section{A OBTENÇÃO DAS DESCRIÇÕES TERMODINÂMICAS}

O comportamento termodinâmico de elementos, substâncias e misturas pode ser medido e representado de um grande numero de formas. A opção CALPHAD, entretanto, é obter uma descrição matemática da energia livre 
de Gibbs, apenas. Em uma visão "matemática" da questão, obter uma descrição da energia livre de Gibbs consiste em encontrar uma função cujos coeficientes se ajustem de modo a reproduzir os dados experimentais com desvio mínimo. Este procedimento é realizado, normalmente, por uma rotina de mínimos quadrados, em um processo chamado "otimização". O processo contempla três etapas e, cada etapa, tem seus riscos e dificuldades.

a) identificar todas as variáveis termodinâmicas que influenciam na energia livre de Gibbs da fase a ser descrita.

De forma geral, se expressa, uma função de pressão, temperatura e composição química, considerando que as propriedades mais complexas (como efeitos magnéticos) podem ser também expressas em função destas variáveis e que não estão presentes outros campos externos;

b) escolher um modelo físico-químico para a relação entre $\mathrm{G}$ e as variáveis termodinâmicas que a influenciam.

Do ponto de vista matemático, um grande número de funções poderia ser empregado para descrever G, dependendo dos dados disponíveis. Do ponto de vista computacional, como as relações entre $G$ e as demais funções termodinâmicas ( $\mathrm{S}, \mathrm{H}, \mathrm{Cp}, \mu$, etc.) envolvem diferenciação e integração, as funções mais convenientes são polinômios relativamente simples

$\mathrm{O}$ aspecto físico-químico, entretanto, é critico, como discutido em detalhe adiante. De forma geral, existe um modelo físico-químico que melhor se ajusta à descrição de $\mathrm{G}$ para cada tipo de fase. A escolha adequada deste modelo resulta em um melhor ajuste, com menos coeficientes; e

c) ajustar os coeficientes da função escoIhida aos dados disponíveis.

$O$ processo de ajuste dos coeficientes é, em geral, um processo que envolve a minimização do desvio entre o valor experimental e o valor calculado para cada condição experimental, numa pratica típica da técnica dos mínimos quadrados. Alguns artifícios importantes são empregados para permitir comparar os desvios entre variáveis tão diferentes como energias, que podem ter valores de $10^{6} \mathrm{~J} / \mathrm{mol}$, temperaturas, freqüente- mente da ordem de $10^{3} \mathrm{~K}$, e atividades, com valores entre 0 e I, como discutido a seguir.

\section{I A Escolha do Modelo Físico-Quimico}

\subsection{Modelo de Solução}

A escolha do modelo físico-químico tem grande importância na capacidade de se obter uma boa descrição matemática para a energia livre de Gibbs de uma fase. De uma forma geral, para uma mistura, a energia livre de Gibbs é expressa como mostra a Equação I:

$\mathrm{G}^{\Phi, \mathrm{T}}={ }^{\mathrm{ref}} \mathrm{G}^{\Phi, \mathrm{T}}+{ }^{\mathrm{conf}} \mathrm{G}_{\mathrm{m}}^{\Phi}+{ }^{\mathrm{xs}} \mathrm{G}_{\mathrm{m}}^{\Phi}$

Onde, o termo com sobrescrito "ref" representa a contribuição para a energia livre dos elementos não misturados; o termo com sobrescrito "conf" a contribuição configuracional para a energia livre (em geral associada à variação de entropia no processo de mistura), obtida diretamente do modelo físico-químico escolhido; e o termo com o sobrescrito "xs", o desvio entre a realidade e o modelo escolhido, a chamada "energia de excesso". Os valores de ${ }^{\text {conf }} \mathrm{G}_{\mathrm{m}}{ }^{\top}$ nem sempre são mostrados explicitamente pelos programas de termodinâmica computacional. Quando o efeito de sua escolha não é levado em conta corretamente, efeitos dramáticos podem ser observados. A Figura I mostra um exemplo da contribuição configuracional para uma solução binária, em função do modelo de solução adotado.

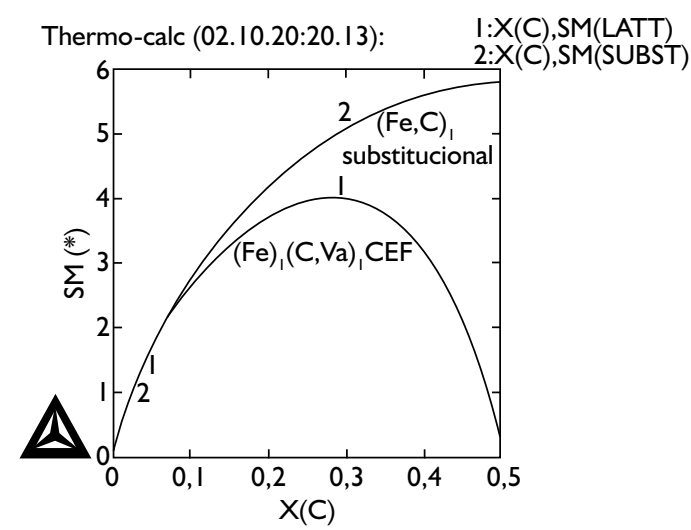

Figura I. Efeito da escolha do modelo de solução sobre a contribuição "configuracional" da energia livre calculada. CEF é o compound energy formalism ou modelo de sub-redes. ${ }^{(7)}$ A descrição adequada para uma estrutura $\mathrm{CCC}$ em que o carbono é intersticial seria a do CEF. Usar a descrição substitucional, neste caso, implicaria em compensar a diferença com um termo de "excesso" ajustado empiricamente.

Em alguns casos, mesmo com um grande esforço de ajuste do termo de excesso, a descrição obtida é pouco adequada, como mostra o exemplo da Figura 2.

Por ocasião da avaliação de sistemas binários, visando obter descrições compatíveis para sistemas de mais alta ordem, é importante que os modelos físico-químicos sejam compatíveis em todos os sistemas binários, para que se possa obter descrições válidas. Um exemplo clássico é o tratamento dos carbonitretos presentes em aços, especialmente microligados e IF. A descrição atualmente aceita é de $(\mathrm{Fe}, \mathrm{Nb}, \mathrm{Ti})$, (C,N, Va), empregando o modelo CEF. 

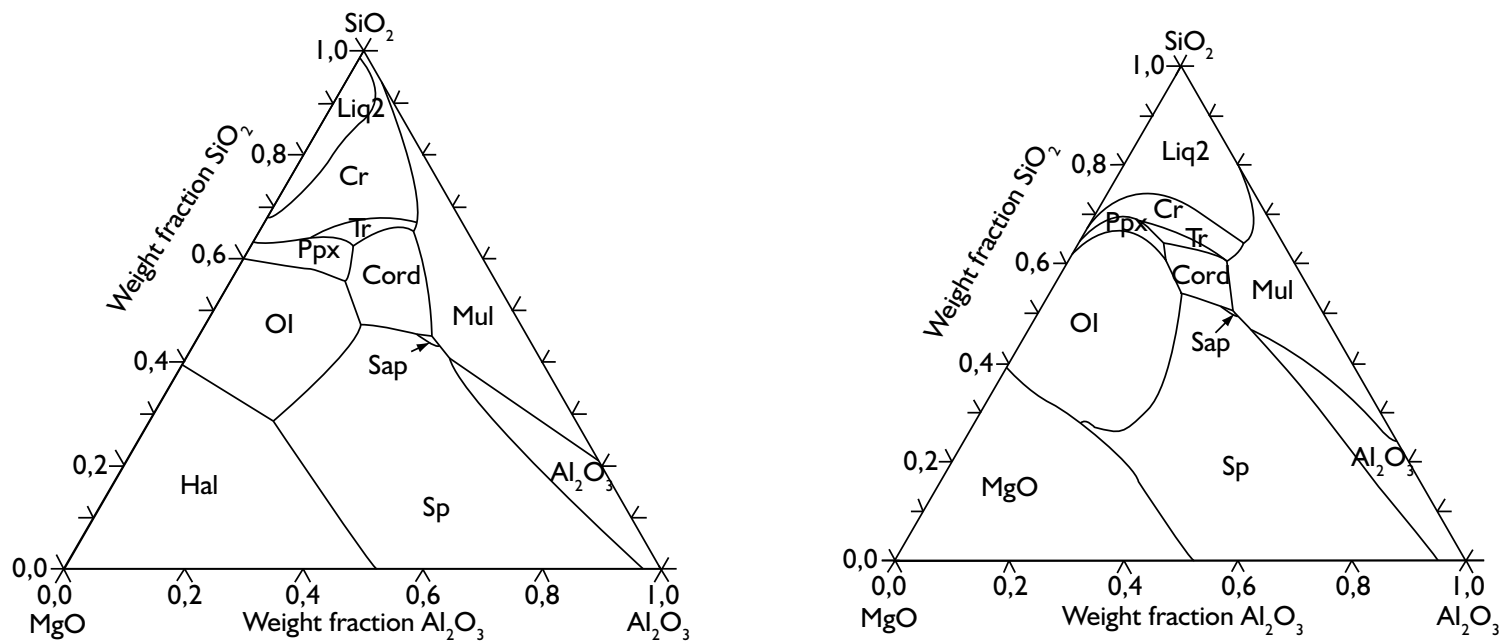

Figura 2. Superfície liquidus do sistema $\mathrm{MgO}-\mathrm{Al}_{2} \mathrm{O}_{3}-\mathrm{SiO}_{2}$ calculada empregando dois modelos diferentes para a energia livre de Gibbs do liquido. Embora o ajuste dos sistemas pseudo-binários individuais seja excelente com os dois modelos, é impossível reproduzir a extensão da imiscibilidade nos líquidos ricos em sílica usando o modelo de líquido iônico ${ }^{(8)}$ (direita), enquanto bons resultados são obtidos com o modelo de Kapoor-Frohberg-Gaye ${ }^{(9)}$ (esquerda). ${ }^{(10)}$

Assim, os binários relevantes precisam adotar este modelo para os compostos em questão.

\subsection{Modelo de Extrapolação de Energia de Gibbs de Excesso}

Quando se calcula a energia de Gibbs de excesso em um sistema de ordem 3 (ternário) ou superior, é necessário escolher um critério para ponderar a contribuição de cada um dos binários (ou sistema de ordem imediatamente inferior). Tradicionalmente, o modelo mais empregado, em programas de termodinâmica computacional, é o modelo de Muggiannu. A discussão sobre o efeito desta escolha é antiga. ${ }^{(1,12)}$ Entretanto, o efeito da escolha ainda é negligenciado, especialmente em sistemas muito assimétricos, como indica o exemplo da Figura 3.

\subsection{A Matemática da Regressão - Otimização}

O uso do termo "otimização", o significado físico dos dados e a complexidade do processo de ajuste por vezes conduzem a erros que não seriam sequer cogitados em outros processos de ajuste estatístico de uma função a um conjunto de dados.

\subsection{As falhas "inerentes" ao método de regressão}

\subsection{I.I O modelo descreve bem os dados?}

O método de regressão busca minimizar a soma dos quadrados dos "erros" isto é, das dife-

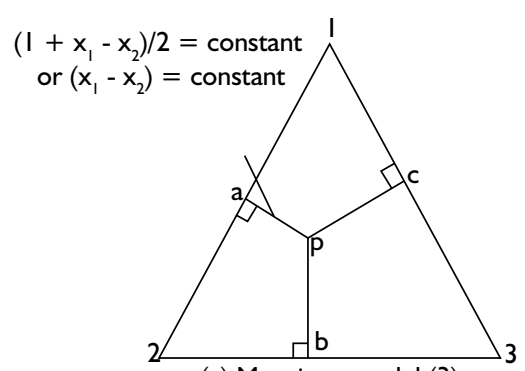

(c) Muggianu model (3)
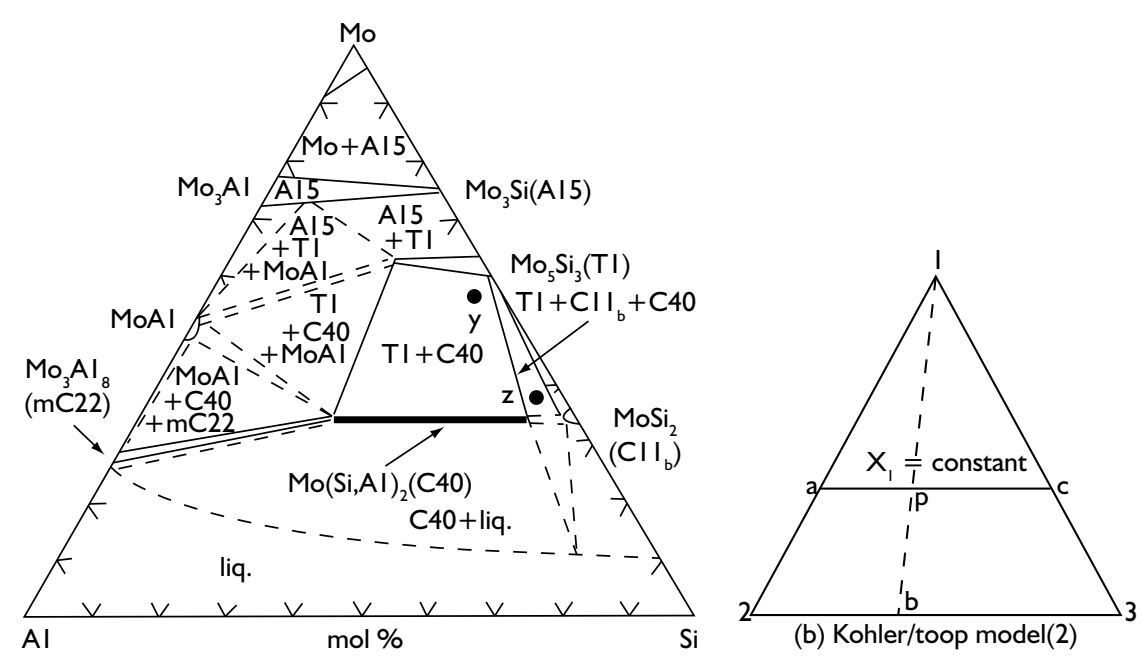

(b) Kohler/toop model(2)

Figura 3. Em um sistema ternário em que há semelhanças entre algumas interações binárias (Mo-Al e Mo-Si, no caso, apresentam interações fortes) e discrepâncias fortes (Al-Si não formam compostos) o método de Kohler (à direita) é mais adequado do que o método de Muggianu (à esquerda) para a energia livre das soluções ternárias, como o líquido, por exemplo. (Pelton ${ }^{(13)}$ apresenta uma discussão interessante sobre o tema). 
renças entre o valor medido e o valor calculado pela equação proposta. Para tal, varia os diversos coeficientes da equação proposta, até encontrar o menor somatório dos quadrados dos "erros". Este é o único critério de "qualidade" do ajuste. Quando a descrição buscada visa interpolar ou extrapolar, 0 ajuste obtido pode ser de pouca utilidade, como mostra a Figura 4.
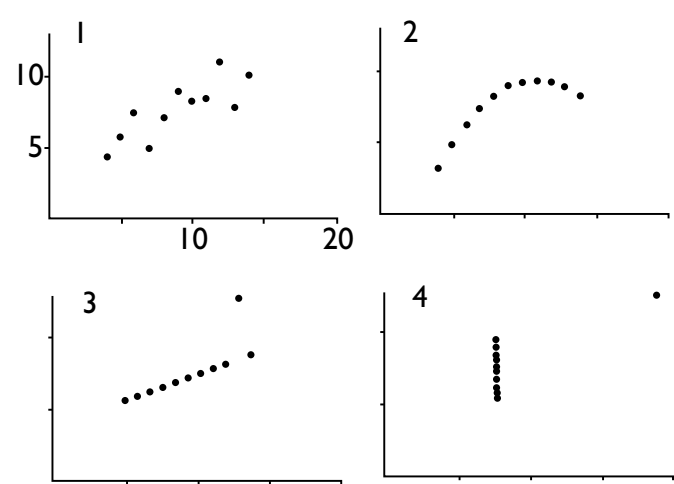

Figura 4. Quando se ajusta uma reta, através de regressão linear, às quatro populações indicadas, obtém-se, como resultado $y=3+0,5 x r^{2}=0,67$. Naturalmente, esta equação não é a melhor maneira de descrever os dados, em alguns dos casos mostrados. ${ }^{(14)}$

Uma análise da qualidade do ajuste, como a exemplificada na Figura 4, não é tão fácil de realizar no caso de uma otimização em termodinâmica computacional, pois a regressão é multi-variada e os dados são de diferentes tipos e, conseqüentemente, diversas magnitudes. Uma análise dos resíduos e ao menos uma verificação de que os dados não estão todos concentrados em uma faixa de temperatura ou de composição química pode evitar dissabores.

\subsection{I.2 Quantos coeficientes?}

Todo estudante de engenharia sabe que - coeficiente de correlação aumenta quando o número de graus de liberdade (pontos experimentais menos coeficientes a ajustar) diminui, até o ponto em que é sempre possível atingir correlação "perfeita" quando se o número de graus de liberdade chega a zero. Infelizmente, a descrição assim obtida raramente é correta, do ponto de vista físico. A incapacidade de resistir à tentação de introduzir coeficientes adicionais é, possivelmente, a maior fonte de dissabores e de avaliações inúteis publicadas na área da termodinâmica computacional.

\subsubsection{A regressão multi-variada de $\mathbf{G}$ - quanto vale cada erro?}

Quando dados experimentais, como uma temperatura de $1000 \mathrm{~K}$ e uma atividade de $0,0 \mathrm{I}$, são incluídos na regressão, qual o critério para comparar o ajuste do cálculo a estes valores? Uma rotina comumente usada, ${ }^{(15)}$ utiliza uma normalização dos dados, baseada na precisão informada para a medida, como mostra a Equação 2. Assim, o erro experimental (ou precisão) atribuído a uma determinada grandeza ou medida tem influência decisiva sobre sua importância para o ajuste dos coeficientes.

Sum of Squares $=\sum\left(\frac{X_{\text {experimental }}-X_{\text {calculado }}}{\text { Erro Experimental }}\right)^{2}=\sum\left(\frac{\Delta}{\varepsilon}\right)^{2}$

\subsubsection{Quais coeficientes?}

Escolhido um modelo físico-químico, a função que descreve a energia livre de Gibbs de excesso deve ser um polinômio. $O$ mais usado, o polinômio de Redlich-Kiester, é um dos mais simples a atender as exigências para consistência das funções termodinâmicas além de permitir fácil manuseio computacional e extrapolação consistente para sistemas de mais alta ordem. A Equação 3 apresenta a forma do polinômio de Redlich-Kiester para um sistema binário.

$$
\begin{aligned}
& \Delta \mathrm{G}_{\mathrm{m}}^{\mathrm{E}}={ }^{\mathrm{E}} \mathrm{G}_{\mathrm{m}}^{\mathrm{T}}=\mathrm{X}_{1} \mathrm{X}_{2} \sum_{\nu=0}^{\mathrm{n}} \mathrm{L}_{\nu}^{\mathrm{T}}\left(\mathrm{X}_{1}-\mathrm{X}_{2}\right)^{\nu} \\
& \mathrm{L}_{\nu}^{\mathrm{T}}=\mathrm{A}_{\nu}+\mathrm{B}_{\nu} \mathrm{T}
\end{aligned}
$$

Como os termos de ordem diferente de zero não são simétricos em relação à composição, cada termo tem um efeito diferente sobre o comportamento da energia livre de excesso em função da composição química (Figura 5). A observação (ou a estimativa) do desvio que determinado sistema apresenta em relação ao comportamento ideal é uma boa orientação sobre quais termos deverão (ou não) ser empregados para descrever a energia livre de excesso. Não se deve, necessariamente, usar todos os coeficientes em seqüência, nem tampouco considerar o efeito da temperatura sobre os coeficientes, se não houver forte indicativo desta necessidade.

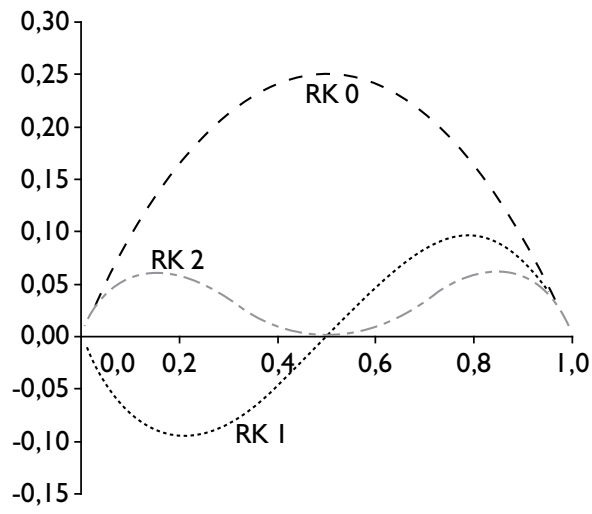

Figura 5. Forma dos três primeiros termos do polinômio de Redlich-Kiester em função da fração molar. Cada termo tem um efeito sensivelmente diferente sobre a energia livre de excesso em função da composição. 


\subsubsection{Interpolação ou extrapolação?}

A inclusão de um número excessivo de coeficientes, especialmente quando se inclui coeficientes que representam variação de $G$ com a temperatura, pode conduzir a resultados inesperados $\mathrm{e}$ a erros significativos. Isto é especialmente relevante quando os dados disponíveis cobrem uma faixa estreita de temperatura, quando aumenta $\circ$ risco de erro nas extrapolações de temperatura (Figura 6).

\subsection{Os Dados Empregados}

A possibilidade de empregar qualquer tipo de dado termodinâmico ou informação de diagrama de equilíbrio de fases como dado de entrada para ajustar uma descrição matemática adequada para a energia livre de Gibbs das fases de um sistema é certamente, uma das maiores forças da termodinâmica computacional. Entretanto, alguns dados são mais valiosos do que outros.

\subsection{Dados de diagramas de fases}

Um diagrama de equilíbrio de fases permite obter informações sobre a relação entre os valores das energias livres de Gibbs

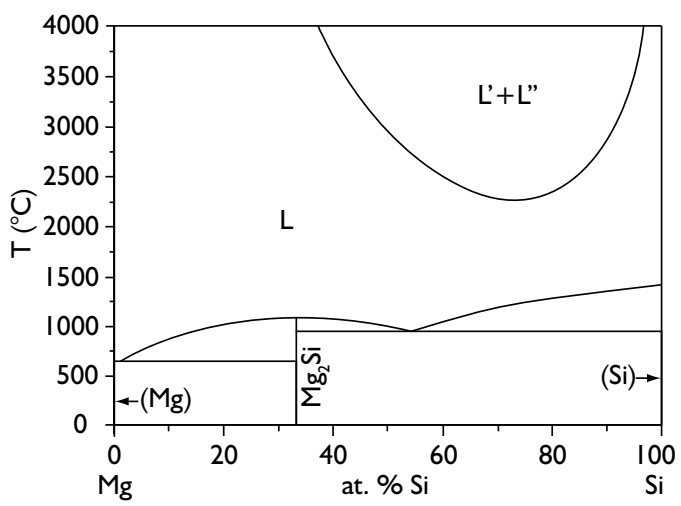

Figura 6. Coeficientes inadequados resultam em uma "imiscibilidade" irreal no líquido. O exemplo é apresentado e discutido por Schmid-Fetzer. ${ }^{(2)}$ É boa prática sempre testar a descrição termodinâmica a temperaturas elevadas e baixas, mesmo que estas não sejam as faixas de interesse "prioritário". das fases presentes no diagrama. Infelizmente, não permite obter qualquer informação sobre os valores "corretos" destas energias. Para cada diagrama de equilíbrio de fases há um numero infinito de combinações de energias livres de Gibbs para as fases em questão que reproduzem o diagrama, como mostra o exemplo simples da Figura 7.

Assim, uma descrição obtida somente com informações de diagramas de fases pode ter utilidade muito limitada. É essencial que alguma informação termodinâmica seja empregada para fixar os valores de $G$.

Ainda assim, tem se observado, na literatura, uma tendência a ajustar as descrições termodinâmicas de modo a reproduzir as temperaturas dos invariantes com exatidão superior a própria precisão das medidas de temperatura. ${ }^{(16)}$

Alguns outros erros importantes podem ser observados quando se privilegia os dados de diagramas de fases sem dispor de dados termodinâmicos. Como se ajusta somente a posição relativa da estabilidade das fases, a solubilidade real pode também ser estimada erradamente.

\section{CONCLUSÕES}

O método CALPHAD é uma poderosa ferramenta para prever o comportamento de equilíbrio em sistemas complexos. $O$ esforço do emprego do método não se justifica como ferramenta para a reconstituição de diagramas simples. A única justificativa é a capacidade de extrapolar para sistemas complexos, com base nos sistemas mais simples. Para tal, é essencial que $o$ ajuste das descrições matemáticas da energia livre de Gibbs das fases envolvidas em tais sistemas seja realizado considerando a) o emprego de um modelo físico-
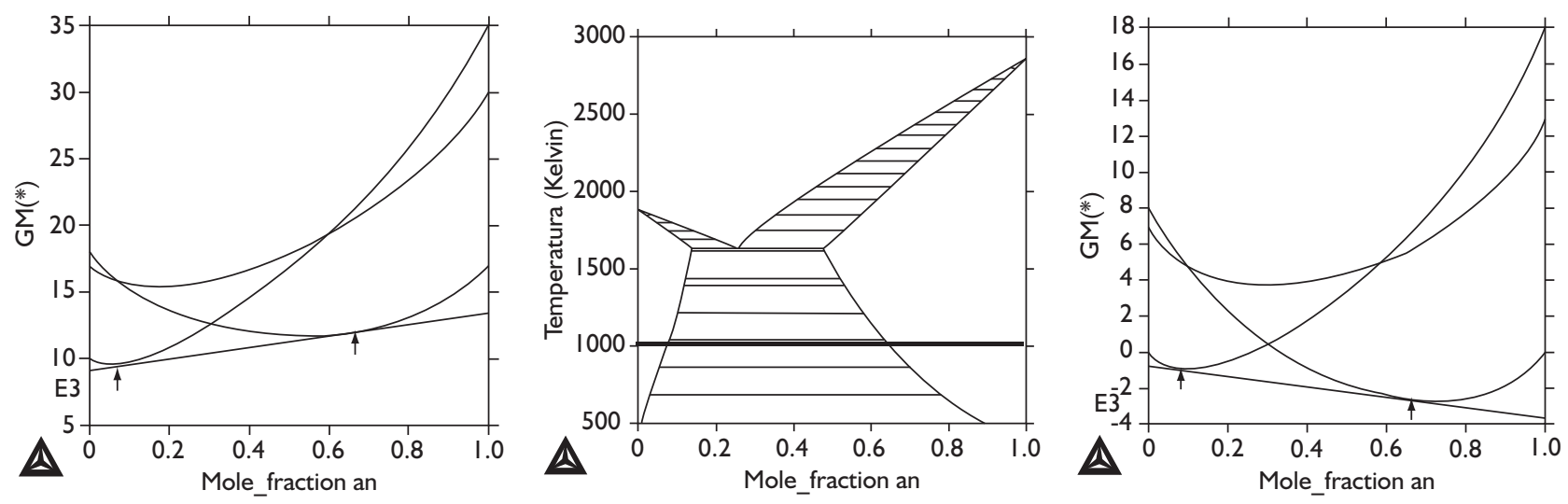

Figura 7. O diagrama da figura central pode ser calculado com duas descrições diferentes para $\mathrm{G}$ das três fases. As figuras a esquerda e a direita mostram curva de $G$ versus composição que resultam no mesmo diagrama para a temperatura indicada pela linha no diagrama. 
químico adequado à fase a modelar; b) dados termodinâmicos confiáveis, além dos dados de diagramas de fases; e c) o menor número possível de coeficientes ajustados.

Embora amplamente empregada como demonstração da qualidade da descrição termo- dinâmica das fases de um sistema, a capacidade de reproduzir um diagrama de fases experimental, mesmo que com "grande precisão" é um teste fraco da qualidade da descrição termodinâmica das fases. Algumas fontes importantes de erro na aplicação do método CALPHAD são apresentadas e discutidas neste trabalho, e formas de evitá-las são apresentadas.

\section{REFERÊNCIAS}

I KUBASCHEWSKI, O. With one auspicious and one dropping eye. CALPHAD, v. 8, n. 4, p. 355-358, Oct.Dec. 1984.

2 SCHMID-FETZER, R.; ANDERSSON, D.; CHEVALIER, P. Y.; ELENO, L.; FABRICHNAYA, O.; KATTNER, U. R.; SUNDMAN, B.; WANG, C.; WATSON, A.; ZABDYR, L.; ZINKEVICH, M. Assessment techniques, database design and software facilities for thermodynamics and diffusion. CALPHAD, v. 3I, n. I, p. 38-52, Mar. 2007.

3 ANSARA, I. Thermodynamic modelling of solution phases and phase diagram calculations. Pure and Applied Chemistry, v. 62, n. I, p. 7I-78, Feb. 1990.

4 KAUFMAN, L.; BERNSTEIN, D. H. Computer calculation of phase diagrams: refractory materials. New York: Academic Press, 1970. v. 4.

5 DINSDALE, A. T., SGTE data for Pure Elements. CALPHAD, v. I5, n. 4, p. 317-425, oct.-dec. 1991.

6 Costa e Silva, A. Representação de dados termodinâmicos de soluções em siderurgia. In: Congresso Anual da ABM, 5I., 1996. Porto Alegre. Anais..., Sao Paulo: ABM, 1996. ICD.

7 HILLERT, M.; STAFFANSSON, L.-I. The regular solution model for stoichiometric phases and ionic melts. Acta Chemica Scandinavica,. v. 24, p. 3618-3626, 1970.

8 HILLERT, M.; JANSSON, B.; SUNDMAN, B.; AGREN, J. A two-sublattice model for molten solutions with different tendency for ionization. Metallurgical Transactions A, v. I6a, n. 2, p. 26I-266, 1985.

9 GAYE, H.; WELFRINGER, J. Modelling of the thermodynamic properties of complex metallurgical slags. In: International Symposium on Metallurgical Slags and Fluxes. 2., 1984. Lake Tahoe, Nevada: TMS-AIME, 1984. p. 357-75.

10 FABRICHNAYA, O.; COSTA E SILVA, A.; ALDINGER, F. Assessment of thermodynamic functions in the MgO-AI2O3$\mathrm{SiO}_{2}$ system. Zeitschrift fur Metallkunde, v. 95, n. 9, p. 793-805, 2004.

II ANSARA, I. Prediction of thermodynamic properties of mixing and phase diagrams in multi-component systems. In: symposium held at Brunel University and the NPL 197I. London. Proceedings... London: Her Majesty's Stationery Office, 1971. p. 403-30.

12 ANSARA, I.; BERNARD, C.; KAUFMAN, L.; SPENCER, P. A comparison of calculated phase equilibria in selected ternary alloy systems using thermodynamic values derived from different models. CALPHAD, v. 2, n. I, p. I-I5, 1978.

I3 PELTON, A. D. A general "geometric" thermodynamic model for multicomponent solutions. CALPHAD, v. 25, n. 2, p. 319-328, June 200I.

14 TUFTE, E. R. The visual display of quantitative information. Cheshire, Conn.: Graphic Press, 1983.

I5 SUNDMAN, B.; JANSSON, B.; ANDERSSON, J.O. The Thermo-Calc databank system. CALPHAD, v. 9, n. 2, p. 153-190, Apr.-June 1985.

I6 FERRO, R.; CACCIAMANI, G.; BORZONE, G. Remarks about data reliability in experimental and computational alloy thermochemistry. Intermetallics, v. II, n. II-I2, p. I08I-94, 2003.

Recebido em: 18/01/07

Aceito em: 28/05/07

Proveniente de: CONGRESSO ANUAL DA ABM, 61., 2006, Rio de Janeiro.

São Paulo: ABM, 2006. 